\title{
Microstructure and evolution of mechanically-induced ultrafine grain in surface layer of AL-alloy subjected to USSP
}

\author{
X. Wu ${ }^{\mathrm{a}, * 1}, \mathrm{~N} . \mathrm{TaO}^{\mathrm{b}}, \mathrm{Y}$. Hong ${ }^{\mathrm{a}}, \mathrm{B} . \mathrm{Xu}{ }^{\mathrm{c}}, \mathrm{J} . \mathrm{Lu}^{\mathrm{d}}, \mathrm{K} . \mathrm{Lu}^{\mathrm{b}}$ \\ ${ }^{a}$ State Key Laboratory of Nonlinear Mechanics, Institute of Mechanics, Chinese Academy of Sciences, Beijing 100080, PR China \\ ${ }^{\mathrm{b}}$ Shenyang National Laboratory for Materials Science, Institute of Metal Research, Chinese Academy of Sciences, Shenyang \\ 110016, PR China \\ ${ }^{\mathrm{c}}$ College of Materials Science Engineering, Taiyuan University of Technology, Taiyuan 030024, PR China \\ ${ }^{\mathrm{d}}$ LASMIS, University of Technology of Troyes, 10000, Troyes, France
}

Received 22 November 2001; received in revised form 8 January 2002; accepted 22 January 2002

\begin{abstract}
Experiments were conducted to investigate the ultrafine-grained (UFG) microstructures in the surface layer of an aluminum alloy 7075 heavily worked by ultrasonic shot peening. Conventional and high-resolution electron microscopy was performed at various depths of the deformed layer. Results showed that UFG structures were introdued into the surface layer of $62 \mu \mathrm{m}$ thick. With increasing strain, the various microstructural features, e.g., the dislocation emission source, elongated microbands, dislocation cells, dislocation cell blocks, equiaxed submicro-, and nano-crystal grains etc., were successively produced. The grain subdivision into the subgrains was found to be the main mechanism responsible for grain refinement. The simultaneous evolution of high boundary misorientations was ascribed to the subgrain boundary rotation for accommodating further strains. Formed microstructures were highly nonequilibratory. (C) 2002 Acta Materialia Inc. Published by Elsevier Science Ltd. All rights reserved.
\end{abstract}

Keywords: Nanocrystalline; Microstructure; Aluminium; Transmission electron microscopy (TEM)

\section{Introduction}

Ultrafine-grained (UFG) materials have attracted significant scientific interest [1-3]. These materials

* Corresponding author. Tel.: +1-216-523-7280; fax: +1216-687-9220.

E-mail address: wuxl_cas@hotmail.com (X. Wu).

${ }^{1}$ Now with Chemical Engineering Department, Cleveland State University, 1960 E24th St, SH455, Cleveland, OH 44115, USA. are structurally characterized by very fine grain size (nano- and submicron-order) and large amount of grain boundary area (and volume). UFG materials have unusual and extraordinary mechanical and physical properties that are fundamentally different from, and often far superior to those of their conventional coarse-grained polycrystalline counterparts.

Severe plastic deformation (SPD) is an effective processing method for the fabrication of various UFG structures by imposing intense plastic strains 
into metals and alloys [4-7]. The production of UFG materials by SPD offers two significant advantages over other techniques such as inert gas condensation, high-energy ball milling, and sliding wear. First, it is possible to produce large bulk samples. Second, these samples are free from any residual porosity and contamination. The resultant microstructures introduced by SPD are substantially grain refined along with high internal stresses and high-energy nonequilibrium boundaries $[8,9]$. Several techniques are now available for producing the requisite high plastic strain of the order of several hundreds of percent, including equal-channel angular pressing (ECAP) [5-7], high pressure torsion (HPT) [9], multipass-coinforge [10], multiaxis deformation [11], and repetitive corrugation and strengthening (RCS) [12].

The understanding of the microstructural evolution mechanism involved in SPD is an essential issue of the research topic having great importance from academic and technological points of view. The mechanism should account not only for the grain refinement but also for the generation of high-angle boundaries with increasing strain. Previous investigations have demonstrated that during repetitive deformation, the grain size refinement is most pronounced at the initial stage of the process, for example low and medium strain, and remain virtually unchanged upon further straining. However, at large strains, boundary misorientations dominate [5-7]. Exposure of deforming surfaces to random and multidirectional deformation could effectively enhance evolution of low-angle boundary misorientations into high-angle ones. Recent studies reported the grain refinement associated with the slip systems and their interactions. Ultrafine dislocation cells enclosed by the $\{112\}$ and $\{110\}$ planes are produced by operation of multi-slip systems during ECAP [7]. The generation of ultrafine subgrains resulted from the piling-up of dislocations along the $\{111\}$ glide planes during RCS [12]. However, the mechanism underlying the grain refinement itself during SPD is less developed to date. Even though most investigations have described changes of the microstructures and mechanical properties, they could not reveal the microstructural response to the dynamic plastic straining and hence, could not clarify the relation of UFG structures to plastic straining.

A SPD based mechanical treatment for surface nanocrystallization (SNC), ultrasonic shot peening (USSP), is recently proposed [13]. It has been demonstrated that during the process of USSP, the UFG structures are produced through introducing intense strains and high strain rates into the surface layers $[14,15]$. The strains present gradient distribution in the surface layer, changing from the maximum at the top surface to zero far into the matrix [16]. The microstructure observation at various depth levels can, therefore, provide the clue to the process of structural evolution relevant to various stages of strain. In the present study, we have investigated the microstructures in the surface layer of an aluminum alloy 7075 heavily worked by USSP through transmission electron microscopy (TEM) and high resolution TEM observation, with the aim at understanding the microstructural evolution and grain refinement mechanism associated with the formation of UFG structures.

\section{Experimental materials and procedures}

\subsection{Material}

The experimental material was a high purity aluminum alloy 7075 , with a composition (wt pct) of $2.5 \mathrm{Mg}, 5.6 \mathrm{Zn}$, and $2.2 \mathrm{Cu}$, balance $\mathrm{Al}$. A commercially available plate was cut into pieces with $100 \times 100 \times 10 \mathrm{~mm}^{3}$ in dimension. A smooth surface finish was attained on the faces by polishing on 700-grade SiC paper. Microscopic examination revealed an initial grain size of the order of $\sim 80$ $\mu \mathrm{m}$.

\subsection{Deformation facility}

The principle of the USSP technique was as follows. A high-energy ultrasonic generator of high frequency $(20 \mathrm{kHz})$ vibrated the reflecting chamber, where the stainless steel shots of $7.5 \mathrm{~mm}$ diameter resonated. The shots then performed repetitive, high-speed, and multi-directional impact onto the surface of materials. Resultantly, severe strains were imparted into the surface by contact 
loading. A strain gradient changing from zero far into the matrix to the maximum at the top surface will simultaneously be established. Details of the equipment were reported in previous articles $[13,14]$. In the present investigation, the USSP processing was conducted under vacuum at room temperature for 15 minutes.

\subsection{Microstructural examination}

Electron microscopy was conducted with a JEOL-2010 transmission electron microscope (TEM) operated at $200 \mathrm{kV}$ for examination of the general microstructural features at low magnifications and a JEM-2010FEF high-spatial-resolution analytical electron microscope (HRTEM) for high magnifications and lattice image observations in grains with a focused beam having a diameter of $\sim 1 \mathrm{~nm}$. Lattice images were taken at close to the optimum defocus conditions, typically at a magnification of $500,000 \times$, with the selected grain oriented close to $\langle 111\rangle$ for lattice imaging.

Thin TEM films were prepared in the sequence of steps, namely, (1) to stick a castolite plate $2 \mathrm{~mm}$ thick on the peened surface, (2) to cut a bar $3 \mathrm{~mm}$ in diameter with the peened layer located at the middle, (3) to cut discs $30 \mu \mathrm{m}$ thick using a diamond saw normal to the long axis of the bar, and (4) to dimple and argon-ion-beam thin to perforation at room temperature. This method could allow the inspection of a well-identified depth of the processed surface layer.

The average boundary misorientation angle, $\gamma$, between neighbouring subgrains was measured with the aid of a TEM double-tilt holder. The method was to focus the electron beam individually on each subgrain, then to adjust the same crystallographic orientation of both subgrains to be parallel to the electron beam, and finally record the angles of both $X(\theta)$ - and $Y(\xi)$-axis for two subgrains, respectively, i.e., $\left(\theta_{1}, \xi_{1}\right)$ and $\left(\theta_{2}, \xi_{2}\right) . \gamma$ was determined as $\gamma=\cos ^{-1}\left[\sin \theta_{1} \sin \theta_{2}+\cos \theta_{1} \cos \right.$ $\left.\theta_{2} \cos \left(\xi_{1}-\xi_{2}\right)\right]$ [17].

The measurements of the grain sizes were made directly from TEM photomicrographs and the reported values were the averages from 40-60 individual measurements. Because of the elongated nature of the subgrains, the datum points were sep- arately presented for measurements of the average of the short axis, the average of the long axis, and the average from randomly selected directions, whereas measurements for the grains were taken consistently along randomly selected directions.

In the present study, the terms subgrain and grain were defined as follows. Areas with a 1 to 2 degree misorientation across the boundaries were called subgrains whereas the term grain became meaningful if there is at least a 6 to 10 degree misorientation across the boundary $[18,19]$. In the case where a continuum of structural states was created (present study), the term should be related to the level of misorientation.

\section{Experimental results}

\subsection{Grain size and depth}

Quantitative measurements of the grain sizes in the affected surface layer are shown in Fig. 1. The grain size refines remarkably into the nanometer regime $(<100 \mathrm{~nm})$ within the outer surface of $\sim 22$ $\mu \mathrm{m}$ thick. The submicro-grained $(0.1-1 \mu \mathrm{m})$ structure is present in the sub-surface of the layer of $\sim 35 \mu \mathrm{m}$ thick. Next to the strain-free matrix is the extended microbands characterized by elongated subgrains $(>1 \mu \mathrm{m})$. The whole depth is $\sim 62 \mu \mathrm{m}$.

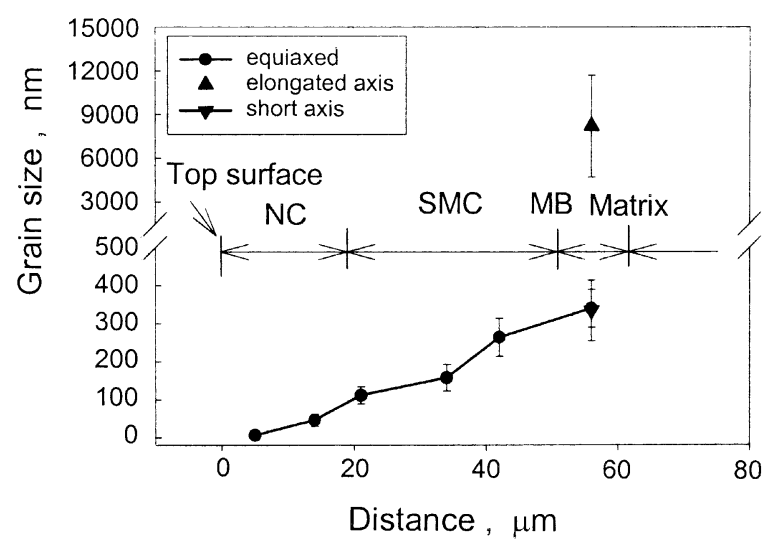

Fig. 1. Variation of grain size with depth of surface layer. 


\subsection{Microbands}

TEM micrographs in Fig. 2 show two prominent deformation features in the area next to the strainfree matrix $(\sim 62 \mu \mathrm{m}$ deep from the top surface) at low strains and would correspond to the initial stage of plastic deformation. First, as shown in Fig. 2(a), the trapezoidal particle of the dispersely distributed second phase could act as an emission source to release a great number of dislocations. The particle is confirmed to be $\mathrm{Al}_{2} \mathrm{Cu}$ based on the analysis of the electron diffraction patterns (EDPs) obtained from the particle. Second, a large number of dislocation cells (DCs) and dislocation tangles are formed inside the interior of the aluminum matrix grains (Fig. 2(b)), responsible for the workhardening during dynamic straining. DCs may transform to individual subgrains upon further plastic straining [12,20-22].

Figure 3(a) shows that the parallel lamellar-type microbands (MBs) of elongated subgrains develop with increasing strain $(\sim 60 \mu \mathrm{m}$ depth from the top surface). Extended MBs are interestingly found to be $\sim 0.6-1.0 \mu \mathrm{m}$ wide but as long as $3-12 \mu \mathrm{m}$. MBs have various orientations, due to the change of the strain path. The EDP (Fig. 3(b)) shows an undeveloped circle with well-defined diffraction spots, indicating that MBs consist of low angle misorientations. For example, the boundary misorientations between subgrains 1 and 2 and between subgrains

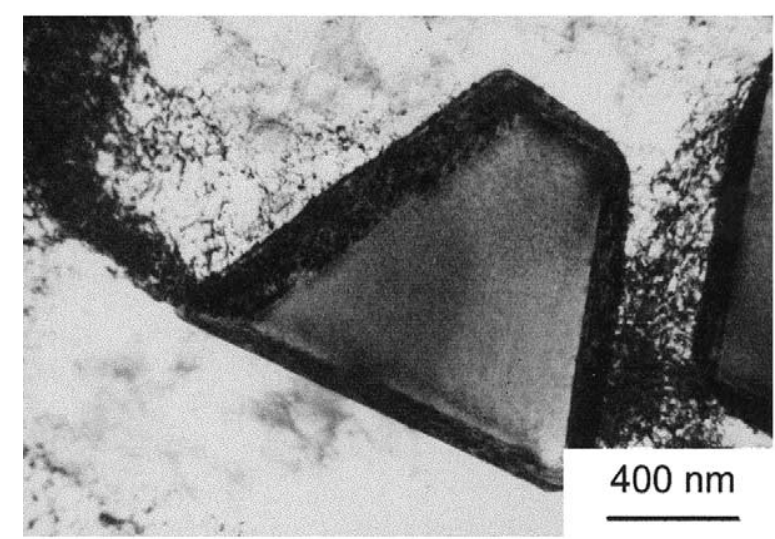

(a)
3 and 4 are determined to be 0.8 and 2.1 degrees, respectively. Such MBs have been observed in previous studies [5-7,18]. The formation of MBs results from the crystallographic slip on a dominant slip system and then propagation by multiple- or cross-slip events because of stress concentration, accompanying the production of a large amount of dislocations between the slip planes $[23,24]$. In view of this, dislocation boundaries and typical lamellar structure with elongated subgrains due to the inhomogeneously distributed dislocations result.

Figure 4(a) shows the further subdivision of MBs at an increased strain $(\sim 58 \mu \mathrm{m}$ depth from the top surface). A large number of cell blocks (CBs) are present inside the original MBs, with very fine dimensions of $\sim 0.2 \mu \mathrm{m}$ wide and $0.4-1.0$ $\mu \mathrm{m}$ long. Figure 4(b) is the EDP with clear brilliant spots as well as some small arcs. It indicates the existence of high angle misorientations among some CB boundaries. With an increase in strain, CBs suffer deformation of various degrees and have to rotate to a certain angle relevant to the deformation direction [25]. The deformation inside each CB is accommodated by one set of slip systems that is different from that in neighbouring CBs.

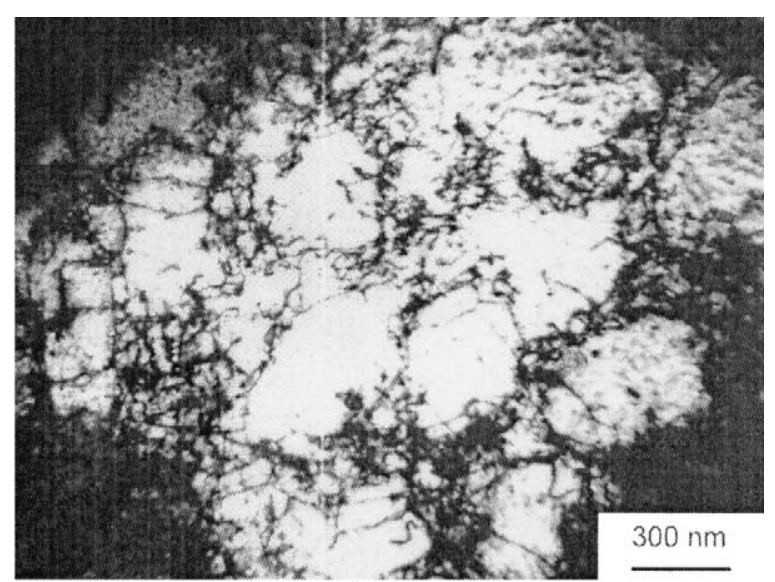

(b)

Fig. 2. TEM micrographs showing: (a) trapezoidal $\mathrm{Al}_{2} \mathrm{Cu}$ particle as emission source of dislocations; and (b) dislocation cells and tanglings in aluminum matrix. 


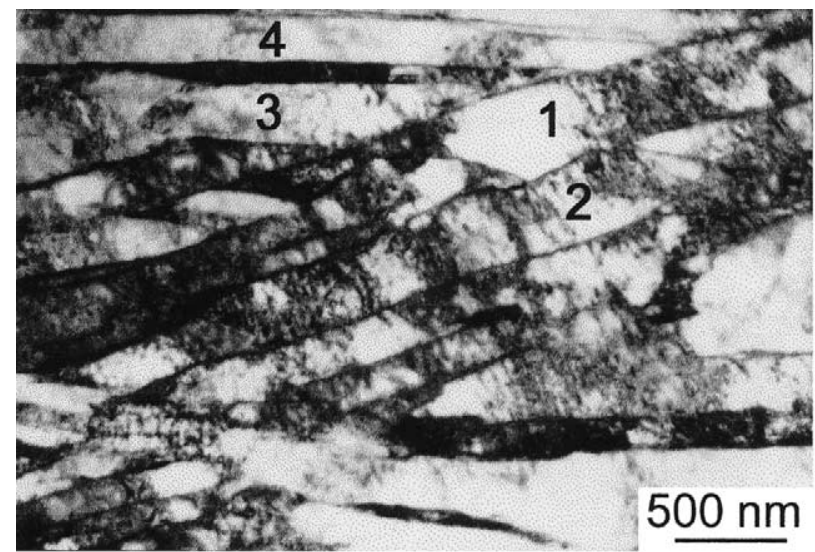

(a)

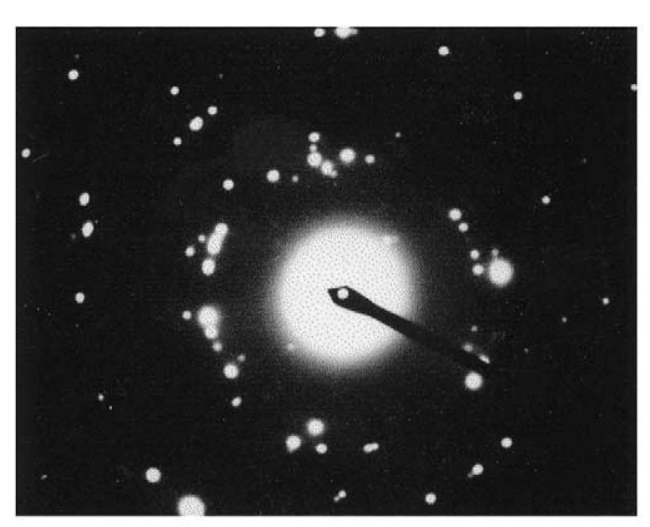

(b)

Fig. 3. (a) TEM micrograph showing lamellar microbands of elongated subgrains; (b) EDP taken from the area covering microbands.

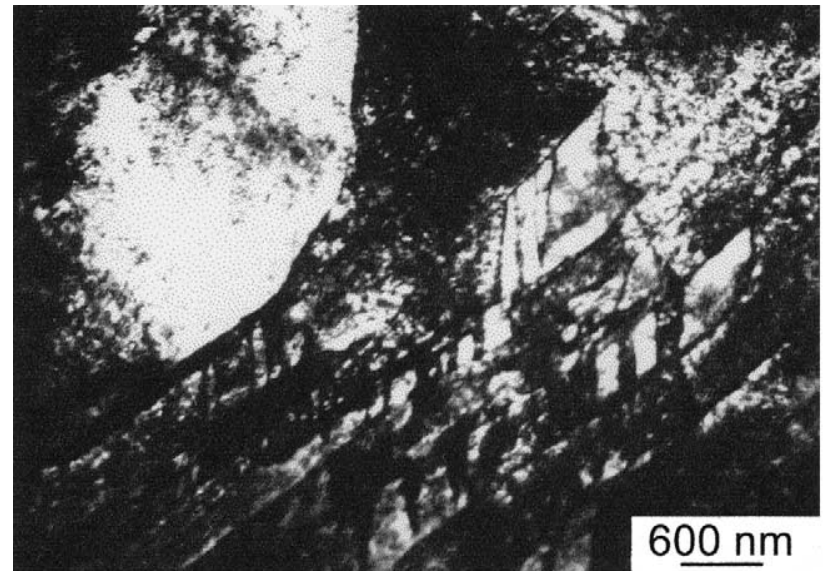

(a)

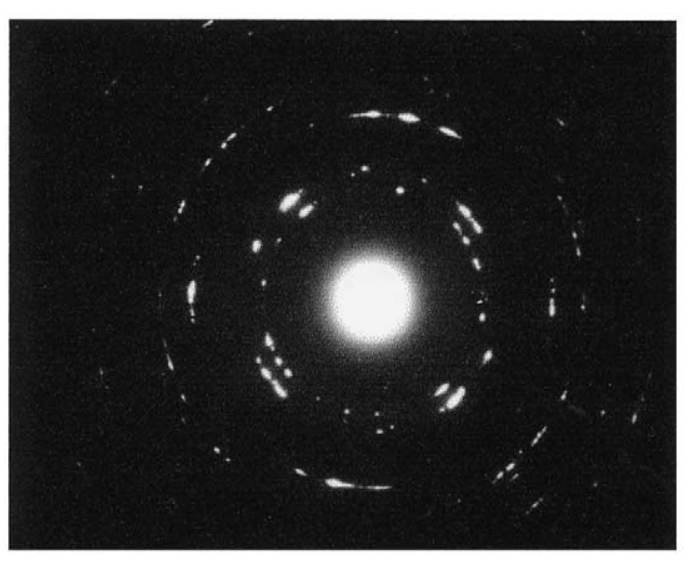

(b)

Fig. 4. (a) TEM micrograph showing microbands with inside dislocation cell blocks; (b) EDP taken from the area covering cell blocks.

\subsection{Submicro-sized microstrucuture}

Figure 5(a) reveals the generation of subgrains inside the interior of a larger grain and the evolution of boundary misorientations at an increased strain $(\sim 50 \mu \mathrm{m}$ deep from the top surface). The grain subdivision leads to the formation of eleven small subgrains of various sizes (denoted by larger size numbers inside the subgrain interior). Boundary misorientations between neighbouring subgrains are determined (denoted by smaller size numbers at grain boundaries). It is interesting to note that three kinds of the boundary morphology encircling subgrain 1 are present. The left side boundary, also a portion of original larger grain, is sharp with a high boundary misorientation of $12.8^{\circ}$. The boundary adjacent to subgrain 11 appears as extinction contours with boundary misorientation of $7.8^{\circ}$, indicating the presence of high internal stresses and non-equilibrium due to the presence of the high density of dislocations at the boundaries [5]. The boundary with subgrain 2 consists of an undeveloped dislocation wall, with boundary misorientation of just $0.8^{\circ}$, and the 

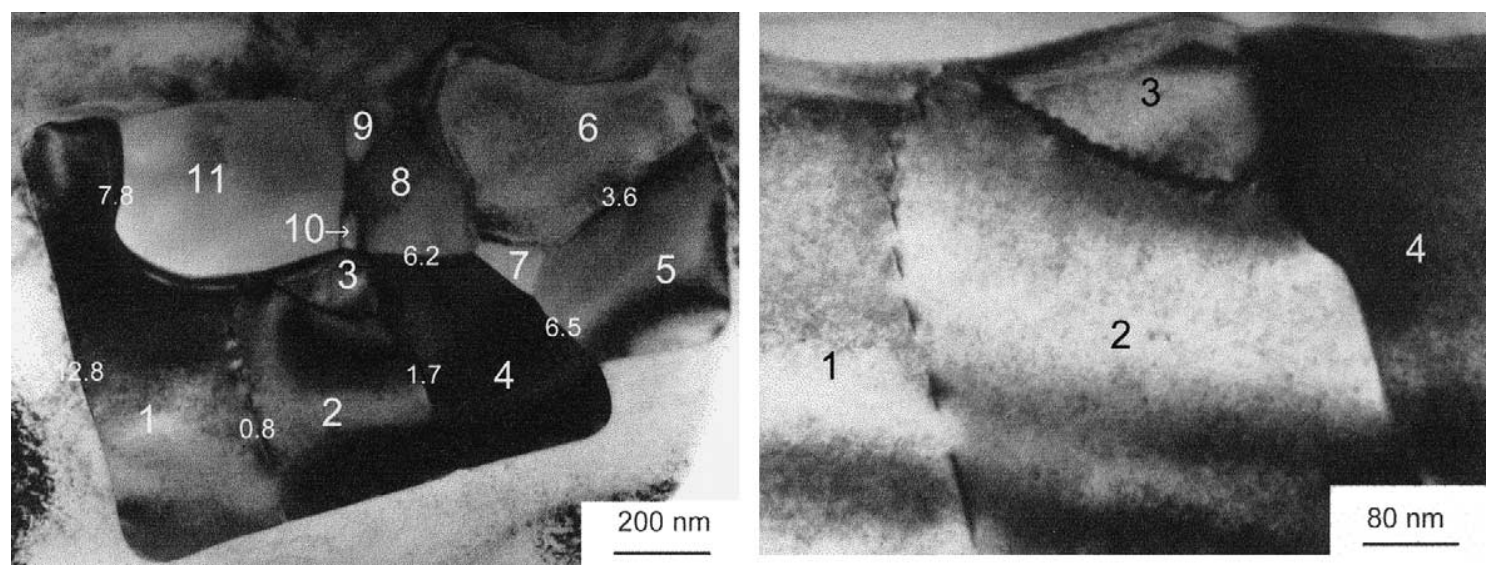

Fig. 5. (a) TEM micrograph showing the formation of subgrains. Large numbers from 1 to 11 denote (sub)grains whereas small ones show boundary misorientation angles between neighboring subgrains; (b) high magnification TEM micrograph showing (sub)grains from 1 to 4 .

boundary can be clearly seen in the high magnification TEM image in Fig. 5(b) along with other grains.

The subgrain boundary evolution has typical history dependence of straining. It is well known that the subgrains form initially with dislocations forming boundaries. After the deformation is completed, no additional dislocations are being added to the boundaries and then the subgrain boundary refinement creates the facet and subsequently, high angle boundaries by reducing the excess dislocation dipoles through dislocation annihilation by dislocation climb [20]. The present observations indicate that the subgrains undergo plastic straining to various degrees, leading to the coexistence of dislocation cells, subgrains, and grains. The dislocation walls should be the subgrain boundary firstly formed by dislocation subdivision upon increased straining. The boundary between subgrains 2 and 3 is also a dislocation wall, just with different dislocation density and spacing. By absorbing dislocations present inside the grain interior, the dislocation wall could evolve into the low angle boundary with an increase in dislocation density and a decrease in dislocation spacing [26]. According to the measured boundary misorientations and morphologies, the sub-boundary, lowand high-angle boundaries are simultaneously present. The low-angle boundary could evolve into the high-angle one upon further plastic straining.
Therefore, the process of the grain refinement consists mainly of the subdivision of grains and the increment in boundary misorientations.

Figure 6 is a TEM micrograph showing the equiaxed, misoriented, and submicro-sized grains. The image is taken at an increased strain $(\sim 40 \mu \mathrm{m}$ deep from the top), as compared with Fig. 5. Inset is the ring-like EDP, indicating that there are many small grains with random misorientations in the selected area of view. Although the grain boundary indi-

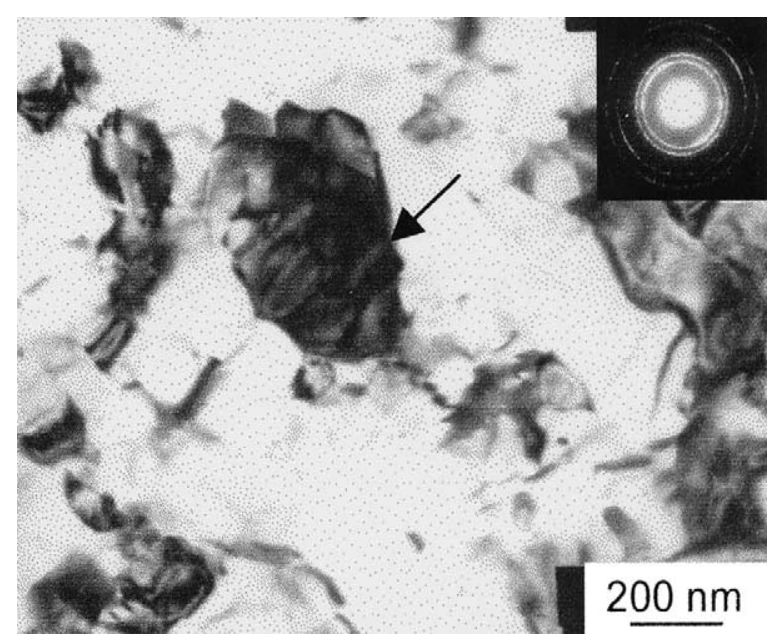

Fig. 6. TEM micrograph showing submicro-grained microstructure. The arrow shows the well-defined subgrain boundary. Inset is EDP. 
cated by an arrow (Fig. 6) is well defined, most grain boundaries are not well defined. However, extinction contours within grains are visible, indicating the presence of high internal stresses and non-equilibrium due to the presence of a high density of dislocations at the boundaries. Similar observations have been reported for UFG structures fabricated by other SPD techniques [5-9].

\subsection{Nanostructure}

Figure 7 is a TEM micrograph illustrating the equiaxed microstructure into the nanometer regime. The image is taken at the outer surface of the layer $(\sim 8 \mu \mathrm{m}$ deep from the top surface). The average grain size is determined to be $\sim 47 \mathrm{~nm}$. Some grain boundaries are visible but many boundaries are poorly defined. Inset is the ring-like EDP indicating highly misoriented boundaries. The submicro- and nano-grained structures obtained in the present investigation have the typical characteristic of nonequilibrium, consistent with those formed using other SPD processes $[8,9,12]$.

Shown in Fig. 8 is another example to depict the grain subdivision and structural evolution with the grain size into the nanometer order $(\sim 4 \mu \mathrm{m}$ deep from the top surface). Figure 8(a) shows two neighboring grains (denoted by 1 and 2 respectively). Figure 8(b) and (c) are their corresponding EDPs. It is interesting to note that the EDP of grain $1(\sim 100 \mathrm{~nm}$ in diameter with clear

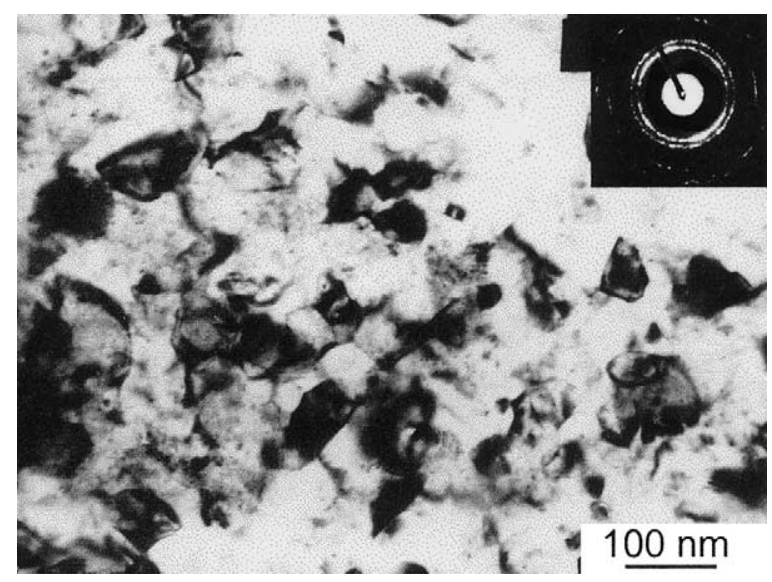

Fig. 7. TEM micrograph showing nanocrystal grains. Inset is EDP. boundary) consists of some well-defined diffraction spots and small arcs, indicating the presence of subgrains. However, the EDP of grain 2 is much different and consists of a set of FCC spots and successive rings, indicating the generation of finer, highly misoriented grains.Figure $8(\mathrm{~d})$ shows the HRTEM image inside the interior of crystal 1. There are many regions, where the two-dimensional lattice fringes are absent, or at best ill defined, suggesting local distortions of crystal lattice, similar to those observed in other UFG materials produced by SPD $[8,9]$. These ill-defined regions subdivide the original grain into subgrains. Figure 8(e) is a HRTEM micrograph showing the presence of several misoriented grains (indicated by arrows) inside the interior of grain 2 . It can be reasonably concluded that the nano-sized subgrains are first produced in both grains and then highly misoriented grains are formed just in grain 2 due to its preferred crystallographic orientation. In other words, the highly misoriented grains come from their precursor subgrains through an increment of boundary misorientations upon straining, the same as in Fig. 5.

\section{Discussion}

\subsection{Subdivision of grains}

Microstructual investigations have revealed that the USSP process can introduce UFG structures in the surface layer of materials. The closer the distance from the top surface of the layer, the finer the grain size, due to the increment of strain over the whole deformed layer [27]. Three levels of grain sizes are present: (1) parallel, extended MBs with interior elongated subgrains and CBs (first level), (2) equiaxed, submicro-grained structure (second level), and (3) equiaxed, nano-grained structure (third level). As the top surface is approached, the grains appear finer, more equiaxed, more misoriented, and more uniformly distributed.

Dislocation gliding, accumulation, interaction, tangling, and spatial rearrangement cause grain subdivision in order to accommodate plastic strains during deformation in polycrystalline materials 

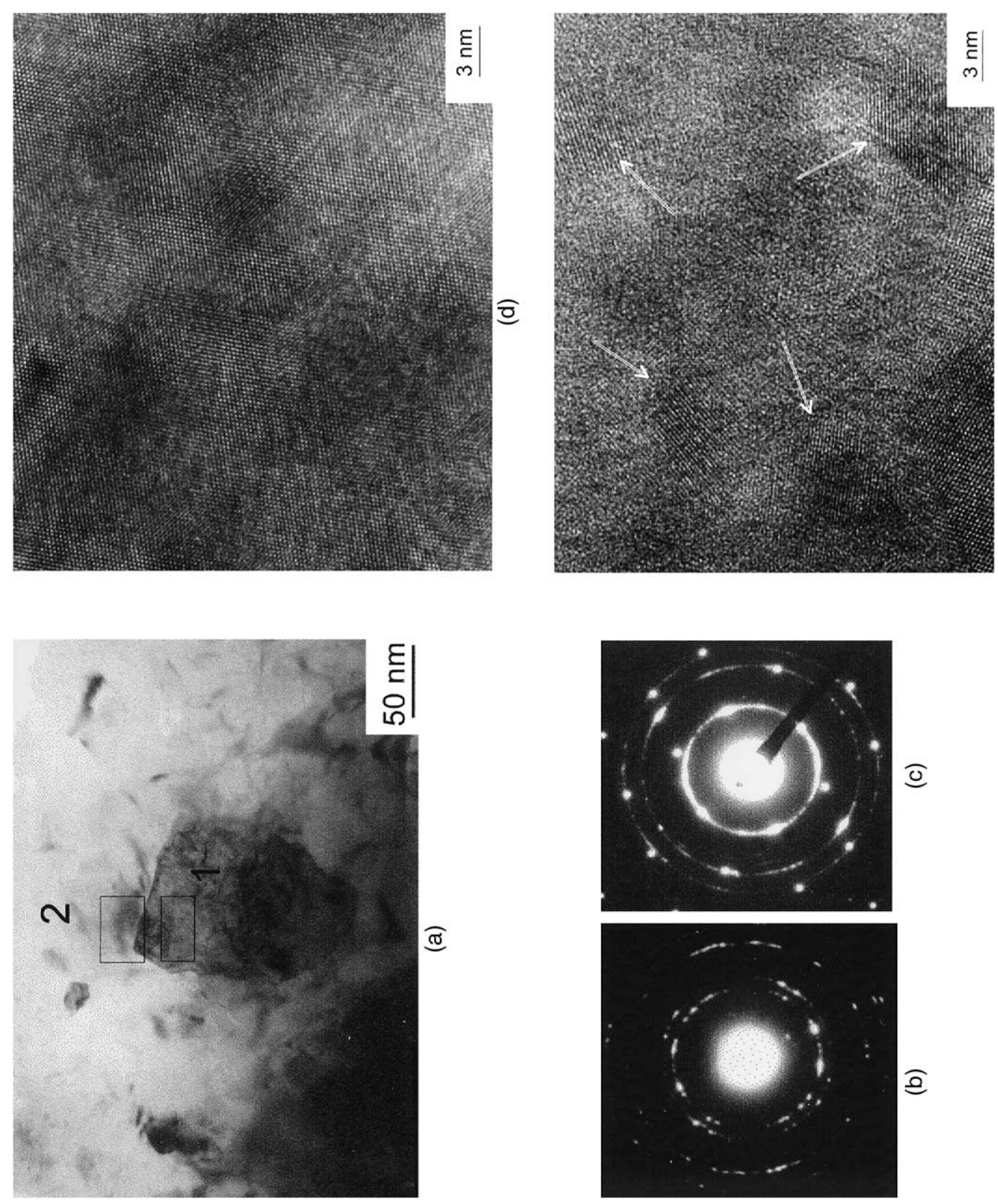

क्)

$\widehat{a}$ 
[20-22]. The repetitive USSP could impart high strains of high strain rates into the surface layer. Severe plastic straining could produce a high density of dislocations (Fig. 2(a)), which are effective at blocking slip at increasing strains and as a result, the mechanism responsible for accommodating large amounts of plastic straining is to subdivide original grains into subgrains with dislocations forming their boundaries. The subdivision of grains takes place on a macroscopic scale with the formation of MBs (Fig. 3(a)) at low strains. With further straining, subgrains may further break up into smaller CBs (Fig. 4(a)). The submicro- (Fig. 5(a)) and nano-sized (Fig. 8(d)) subgrains could be produced under much larger strains.

By performing repetitive USSP, very high strains may be achieved. Note that dislocations of high density are always present in deformed structures of various grain sizes (Figs 3(a), 4(a), 5(a), and $8(\mathrm{~d})$ ). Simultaneously, low angle boundaries are produced (Figs 5(a) and 8(d)), which means new boundaries are being continuously formed during the deformation. With increasing strain, the microscopic subdivision takes place on a finer and finer scale. Resultantly, the process of structural refinement could conduct successively into submicro- (Figs 5 and 6) and nano-meter (Figs 7 and 8) regime.

The multi-directional peening may lead to the change of slip systems with the strain path even inside the same subgrain, much different from the deformation mode caused by other SPD processes. The dislocations not only interact with other dislocations in the current active slip systems, but also interact with inactive dislocations generated in previous deformation. This will promote the formation of subgrains. As a consequence, the effectiveness of grain refinement is enhanced [28].

\subsection{Evolution of highly misoriented boundaries}

The development of equiaxed, highly misoriented grains consists of two steps, i.e., the formation of subgrains through grain subdivision and the subsequent evolution of boundary misorientations. The subgrains resulted from the grain subdivision, however, have a critical size before leveling off, relevant to a certain value of straining [5-7]. The grain subdivision does not continue indefinitely, and eventually, after a given amount of deformation, the continued straining can no longer reduce the subgrain size. At this stage, since the dislocation movement is more strongly restricted, slip systems within adjacent subgrains will be activated in response to applied straining in order to rotate those subgrains into a more energetically favourable orientation [29]. Shot peening provides the multi-directional strain path and high strain rate, which are especially effective at promoting subgrain rotation [27]. The mechanism for the development of high misorientations should be the subgrain rotation. Therefore, the accumulated rotation of subgrains appears to be the primary mechanism as a means of accommodating further deformation, resulting in highly misoriented, equiaxed grains (Figs 5(a), 8(d) and 8(e)).

Deformation of subgrains, as shown in Figs 5 and 8 , is controlled by the activation of slip systems, where the critical resolved shear stress has been achieved. During plastic straining, different slip system combinations would be activated in each individual subgrain. Adjacent misoriented subgrains will have different activated slip systems because of their different orientations. Certain slip systems will be selectively activated to minimize the internal energy in the subgrain. The adjacent misoriented subgrains will rotate into coincidence to minimize the energy across the sub-boundaries under the driving force of the selectively activated slip systems [30]. With increasing strain, subgrains can no longer accommodate deformation by dislocation glide along the same slip systems and, therefore, begin to rotate independently. The rotation angles increase, eventually becoming highly misoriented grains.

As compared with other SPD processing methods, USSP produces a high strain rate, which plays a significant role in lattice rotation during deformation. The high strain rate results in significantly higher flow stresses for an equivalent increment in strain relative to low strain rates. Computer simulation revealed that the higher strain rates promote lattice rotation in simple shear to a greater extent than lower strain rates due to the reduced plastic spin component and the great number of activated slip systems [31]. It is observed 
that the average misorientation angle between the subgrains increased for the same strain, with an increase in strain rate from $6 \times 10^{-6}$ to $6 \times 10^{1} \mathrm{~s}^{-1}$ during tension of pure aluminum [32].

\section{Conclusions}

1. USSP provides a simple and effective procedure for producing a UFG structure on the surface layer of aluminum alloy 7075 .

2. The development of microstructures during the USSP process is characterized by the sequence of elongated microbands (MBs) with dislocation cells (DCs), equiaxed submicro- and nanograins, respectively, with increasing straining.

3. The grain refinement and microstructural evolution during the process of USSP is as follows. During plastic straining, the formation of subgrains through grain subdivision occurs in order to accommodate the strain. The highly misoriented boundaries are generated by the subgrain rotation for accommodating further deformation.

\section{Acknowledgements}

This research was supported by the National Natural Science Foundation of China, National Outstanding Youth Scientific Award of China, and The Chinese Academy of Sciences. One of the authors (X. W.) would like to acknowledge Professor Y. Bai (IM) and Dr L. Lu (IMR) for useful discussions. The assistance of $\mathrm{Mr} \mathrm{X}$. Yong and $\mathrm{Dr}$ G. Liu in conducting USSP processing is greatly appreciated. We would also like to thank the reviewers and editor for valuable comments and suggestions.

\section{References}

[1] Gleiter H. Prog Mater Sci 1989;33:223.

[2] Suryanarayana C. Int Mater Rev 1995;40:41.

[3] Lu K. Mater Sci Eng R 1996;16:161.
[4] Valiev RZ, Korznikov AV, Mulyukov RR. Mater Sci Eng A 1993;168:141.

[5] Iwahashi Y, Wang Y, Horita J, Nemoto M, Langdon TG. Acta mater 1998;46:3317.

[6] Iwahashi Y, Horita Z, Nemoto M, Langdon TG. Acta mater 1997;45:4733.

[7] Shin DH, Kim I, Kim J, Park K. Acta mater 2001;49:1285.

[8] Horita Z, Smith DJ, Furukawa M, Nemoto M, Valiev Z, Langdon TG. J Mater Res 1996;11:1880.

[9] Horita Z, Smith DJ, Nemoto M, Valiev Z, Langdon TG. J Mater Res 1998;13:446.

[10] Ghosh AK, Huang W. In: Lowe TC, Valiev RZ, editors. Investigations and Applications of Severe Plastic Deformation. NATO Science Series, Series 3, High Technology. Boston: Kluwer Academic, 2000;80: 29.

[11] Chen W, Ferguson D, Ferguson H. In: Mishra RS, Semiatin SL, Suryanrayana C, Thadhani NN, Lowe TC, editors. Ultrafine Grain Materials. Warrendale, PA: TMS; 2000. p. 23-5.

[12] Huang JY, Zhu YT, Jiang H, Lowe TC. Acta mater 2001;49:1497.

[13] Lu K, Lu J. J Mater Sci Technol 1999;15:193.

[14] Tao NR, Sui ML, Lu J, Lu K. Nanostru Mater 1999; 11:433.

[15] Liu G, Lu J, Lu K. Mater. Sci. Eng. A 2000;286:91.

[16] Wu X, Hong Y, Lu J, Lu K. In: Meng WJ, Kumar A, Doll GL, Chang YT, editors, MRS Proceedings, Vol. 697, Surface Engineering 2001-Fundamentals and Applications. MRS, 2002, pp. 8.141-8.146.

[17] Chen S, Wang Y. In: Analysis of Electron Microscopy in Materials Science. Beijing: Machine Press; 1995. p. 14-5.

[18] Ferrasse S, Segal VM, Hartwig KT, Goforth RE. Metall Mater Trans A 1997;28:1047.

[19] Andrade U, Mayer MA, Vecchio KS, Chokshi AH. Acta mater 1994;42:3183.

[20] Hansen N. Mater Sci Technol 1990;6:1039.

[21] Liu Q, Juul Jensen D, Hansen N. Acta mater 1998;46:5819.

[22] Hansen N, Huang X, Hughes DA. Mater Sci Eng A 2001;317:3.

[23] Bai YL. J. Mech. Phys. Solids 1982;30:195.

[24] Bai YL, Dodd B. In: Adiabatic Shear Localization: Occurrence, Theories and Applications. Oxford, UK: Pergamon Press; 1992. p. 8-.

[25] Xu YB, Bai YL, Xue Q, Shen LT. Acta mater 1996;44:1917.

[26] Gil Sevillano J, van Houtte P, Aernoudt E. Progress in Mater 1980;25:69.

[27] Wagner L, Lutjering G. In: Niku Lari A, editor. Shot Peening. Oxford, UK: Pergamon Press; 1982. p. 45-3.

[28] Zhu YT, Lowe TC. Mater Sci Engng A 2000;291:46.

[29] Flaquer J, Gil Sevillano J. J. Mater. Sci 1984;19:423.

[30] Bay B, Hansen N, Hughes DA, Kuhlmann-Wilsdorf D. Acta metall mater 1992;40:205.

[31] Canova GR, Fressengeas C, Molinari A, Kocks UK. Acta mater 1988;36:1961.

[32] Korbel A, Swiatkowski K. Met Sci J 1972;6:60. 